\title{
HISTOLOGÍA DE LAS MEMBRANAS EXTRAEMBRIONARIAS DURANTE LA RETENCIÓN INTRAUTERINA EN SCELOPORUS AENEUS (SQUAMATA: PHRYNOSOMATIDAE)
}

\author{
Melody Castaños ${ }^{1}$, Maricela Villagrán ${ }^{2}$, Felipe RodrígueZ1, \\ Fausto MÉNDEZ ${ }^{3}$, Oswaldo HERnández ${ }^{1}$ y Andrés MARTínez ${ }^{1}$ \\ 1 Centro de Investigación en Recursos Bióticos, Universidad Autónoma del Estado de México. Insti- \\ tuto Literario Núm. 100, Col. Centro. C.P. 50000 Toluca, Estado de México. MÉXICO. \\ e-mail: melody_1281@yahoo.com.mx \\ 2 Laboratorio de Biología de la Reproducción Animal, Facultad de Ciencias, Universidad Nacional \\ Autónoma de México. C.P. 04510, Distrito Federal, MÉXICO. \\ 3 Instituto de Biología. Universidad Nacional Autónoma de México. A. P. 70-153, C.P. 04510, \\ México 20, Distrito Federal, MÉXICO.
}

Castaños M. M., M. Villagrán, F. Rodríguez, F. Méndez, O. Hernández y A. Martínez. 2009. Histología de las membranas extraembrionarias durante la retención intrauterina en Sceloporus aeneus (Squamata: Phrynosomatidae). Acta Zoológica Mexicana (n. s.), 25(2): 303-314.

RESUMEN. Se describió la morfología de las membranas extraembrionarias de la lagartija Sceloporus aeneus a partir del estadio de desarrollo embrionario normal a la oviposición (30-31) hasta estadios avanzados de retención (37) utilizando la microscopía de luz. En el estadio normal a la oviposición, la membrana coriovitelina está presente como en otras especies ovíparas, además es reemplazada por la membrana corioalantoidea. En los estadios 34-37, la membrana alantoidea se extiende más allá del ecuador del huevo donde contacta a la onfalopleura bilaminar, sólo en dos especimenes de los estadios 34 y 35, esta membrana rodea por completo al saco vitelino. Durante la retención intrauterina, se observó una membrana corioalantoidea regionalmente diversificada, teniendo un epitelio coriónico escamoso rodeando el hemisferio embrionario y un epitelio cúbico a cilíndrico cubriendo el abembrionario. La onfalopleura bilaminar mostró un epitelio cúbico a cilíndrico en la mayoría de los embriones de estadios avanzados (35-37). Sugerimos que la membrana corioalantoidea juega un papel importante en el transporte de calcio y agua hacia el embrión, ésta última sin provocar el incremento en el volumen del huevo dentro del útero. La persistencia de la onfalopleura bilaminar en estadios de desarrollo avanzados, puede desempeñar la misma función de transporte de agua y calcio durante el intervalo de retención intrauterina, como ocurre en las membranas extraembrionarias de especies vivíparas dentro de la clase Reptilia.

Palabras clave: Histología, membranas extraembrionarias, retención intrauterina, Sceloporus aeneus, viviparidad.

Recibido: 21/02/2008; aceptado: 26/01/2009. 
Castaños M. M., M. Villagrán, F. Rodríguez, F. Méndez, O. Hernández \& A. Martínez. 2009. Histology of extraembryonic membranes during extended egg retention in Sceloporus aeneus (Squamata: Phrynosomatidae). Acta Zoológica Mexicana (n. s.), 25(2): 303-314.

ABSTRACT. Morphology of extraembryonic membranes in the lizard Sceloporus aeneus was described from the modal time of oviposition (30-31) to advanced stages of retention (37) using light microscopy. At the normal stage of oviposition the choriovitelline membrane is present as in other oviparous species, moreover is replaced by the chorioallantoic membrane. At stages 34-37, the allantoic membrane extend beyond the equator of the egg where it contacts the bilaminar onphalopleure. Only in two specimens of stages 34 and 35, this membrane fully encircles the yolk sac. During egg retention, a regionally diversified chorioallantoic membrane was observed, having a thin squamous chorionic epithelia enveloping the embryonic hemisphere, and a cubic or cylindrical epithelia covering the abembryonic one. The bilaminar onphalopleure showed a cubic and cylindrical epithelia in the most advanced embryonic stages (35-37). We suggest that the chorioallantoic membrane plays a role in calcium and water transport towards the embryo, the later not causing any increase in volume of the egg inside the uterus. The persistence of the bilaminar onphalopleure in advanced stages of development may accomplish the same function of water and calcium transport during the interval of uterine egg retention, as it occurs in extraembryonic membranes of viviparous species within Reptilia.

Key words: Histology, extraembryonic membranes, intrauterine egg retention, Sceloporus aeneus, viviparity.

\section{INTRODUCCIÓN}

Los estudios sobre la descripción histológica de las membranas extraembrionarias en reptiles ovíparos son escasos (Habrowski 1926, Stewart 1985, Florian 1990, Stewart \& Thompson 1996, Stewart \& Florian 2000, Stewart \&Thompson 2004). El conocimiento que se tiene acerca de la estructura y función de estas membranas proviene de investigaciones realizadas en escamados vivíparos (para revisión ver Blackburn 1993, Stewart 1993, Stewart \& Thompson 2000, Thompson et al. 2000).

En escamados ovíparos, las membranas extraembrionarias juegan un papel muy importante en el intercambio fisiológico entre el embrión y el ambiente que los rodea (Blackburn et al. 2003). Dentro de algunas de las funciones que desempeñan estas membranas están el intercambio gaseoso y la toma de agua llevada a cabo por el alantoides, así como la provisión de nutrientes a través de los vasos sanguíneos del saco vitelino (Goin et al. 1978).

Se cree que el incremento en la densidad vascular del oviducto y del corioalantoides (placentación sencilla) (Andrews 1997; Andrews \& Mathies 2000), así como especializaciones de tipo hormonal (Guillette 1993) y la reducción en la membrana de la cáscara (Blackburn 1993), permiten la extensión del periodo de desarrollo embrionario dentro del útero (Mathies \& Andrews 2000).

Dado que se tienen pocas descripciones histológicas de las membranas extraembrionarias en reptiles ovíparos, se eligió a Sceloporus aeneus, una especie que tiene la capacidad de retener los huevos hasta estadios embrionarios avanzados (Andrews 1997), para describir los cambios que suceden en estas membranas durante la retención intrauterina. 


\section{MATERIAL Y MÉTODOS}

Se recolectaron 25 hembras gestantes de Sceloporus aeneus durante los meses de abril-julio de 2007 de la localidad el Mapa, Nevado de Toluca, Estado de México $\left(19^{\circ} 11^{\prime} 53^{\prime \prime} \mathrm{N}, 99^{\circ} 50^{\prime} 49^{\prime \prime} \mathrm{O}\right)$ a $3200 \mathrm{~m}$ de altitud. Se colocaron dos hembras en cada terrario de plástico $(30 \times 25 \times 13 \mathrm{~cm})$. Los terrarios estuvieron provistos de vegetación y rocas para asemejar el ambiente donde habitan estas lagartijas. Las hembras fueron alimentadas tres veces por semana con moscas, lombrices, gusanos de seda, grillos y larvas de tenebrio. La mayoría de las hembras fueron puestas en un substrato seco ( $5 \mathrm{~cm}$ de tierra para jardín) para promover la retención de los huevos (Mathies \& Andrews 1996). Se proporcionó agua a las lagartijas mediante aspersión en las paredes de los terrarios y en la vegetación, teniendo cuidado de no mojar el suelo. Tres hembras se mantuvieron bajo condiciones control (substrato húmedo) para establecer el estadio normal de oviposición, para ello se humedeció el suelo con suficiente agua para proporcionar un sitio adecuado para la oviposición (Mathies \& Andrews 1996). El suelo se revisó dos veces al día. Una vez que los huevos fueron ovipositados o extraídos quirúrgicamente de los oviductos, se fijaron en formol al 10\%. Los embriones fueron categorizados de acuerdo a los estadios de desarrollo descritos por Dufaure \& Hubert (1961). Los huevos fueron tratados con la técnica histológica convencional, embebidos en paraplast, cortados en serie a $7 \mathrm{~m}$ y teñidos con hematoxilina-eosina (Humason 1981). La histología de las membranas extraembrionarias se analizó utilizando microscopía de luz y se definieron de acuerdo con Stewart \& Florian (2000). Las fotomicrografías fueron tomadas con una cámara digital Olimpus C5050, utilizando un microscopio Olimpus CX31.

\section{RESULTADOS}

El análisis histológico permitió identificar las siguientes estructuras.

Membrana coriovitelina. En $S$. aeneus la membrana coriovitelina es reemplazada por la membrana corioalantoidea en el estadio 30 (Fig. 1A). Esta membrana presentó un ectodermo coriónico escamoso (Fig. 1B).

Onfalopleura bilaminar. La histología de la onfalopleura bilaminar en esta especie experimenta diversos cambios a través del desarrollo embrionario, principalmente en el ectodermo coriónico que la rodea. Del estadio 29 al estadio 33, el ectodermo es escamoso (Fig. 2A). En algunos embriones del estadio 33 al 35, el ectodermo se vuelve cúbico (Fig. 2B), aunque en algunos embriones de éste último estadio, el epitelio fue cilíndrico (Fig. 2C). Para los estadios 36 y 37, la onfalopleura bilaminar se pliega y todavía ocupa una gran área del hemisferio abembrionario (Figs. 2D y E). En la región central de esta membrana, se presentó una capa sencilla de células de forma cilíndrica que no formó parte de dicho plegamiento (Fig. 2D). 

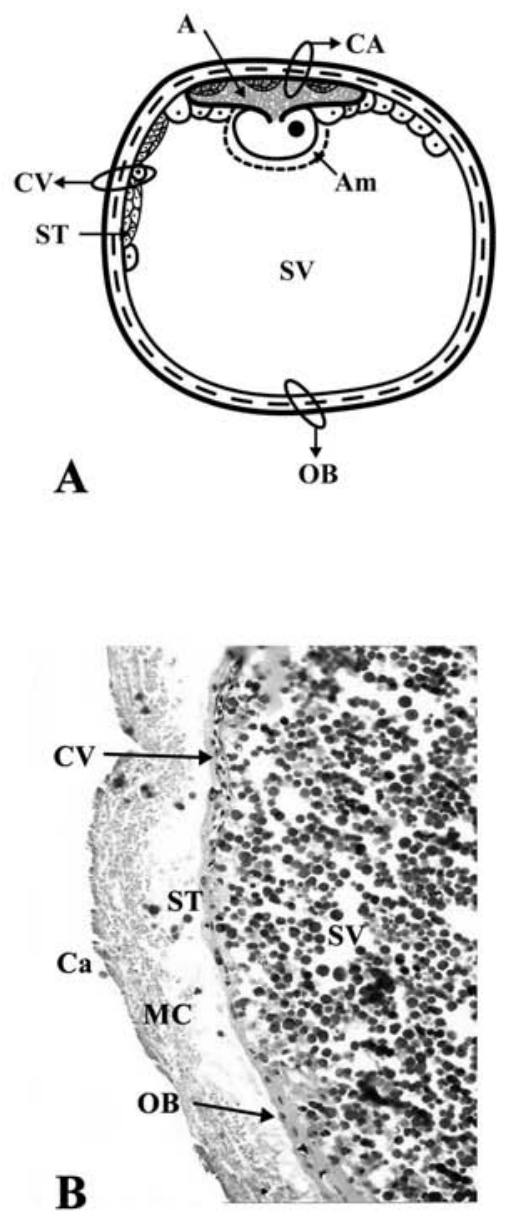

Figura 1. Membranas extraembrionarias en estadio 29. A) Esquema de las membranas extraembrionarias. (A) Alantoides, (Am) Amnios, (CA) Membrana Corioalantoidea, (CV) Membrana Coriovitelina, (OB) Onfalopleura bilaminar, (SV) Saco Vitelino, (ST) Sinus terminalis.

B) Microfotografía de la membrana coriovitelina a nivel del sinus terminalis. (Ca) Calcio de la membrana de la cáscara, (MC) Membrana de la Cáscara, (CV) Membrana Coriovitelina, (OB) Onfalopleura Bilaminar, (SV) Saco Vitelino, (ST) Sinus terminalis. 200x. 

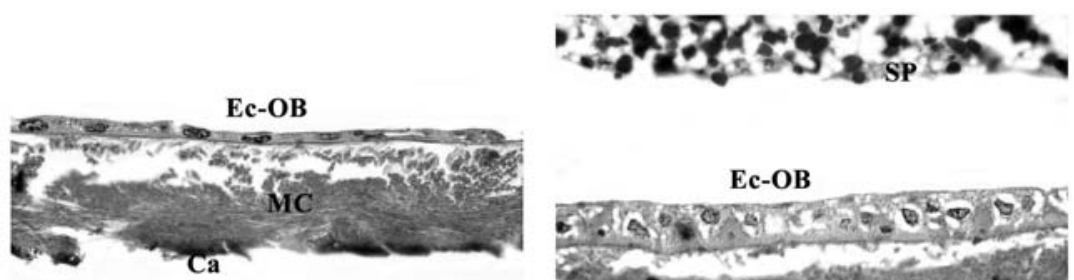

\section{$\mathbf{A}$}
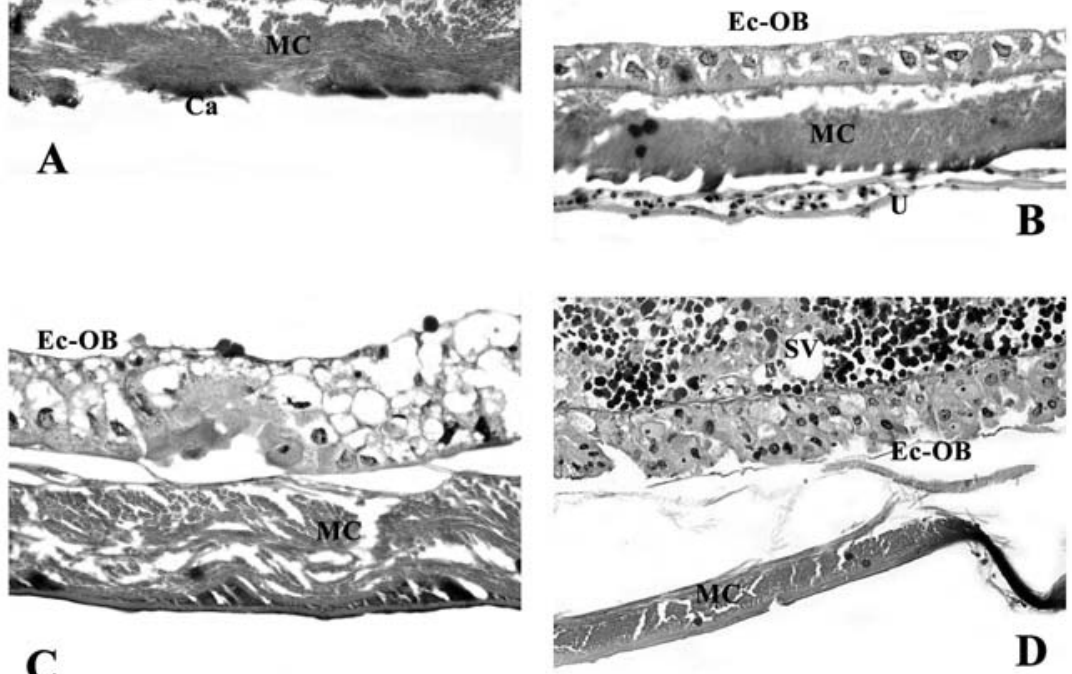

C

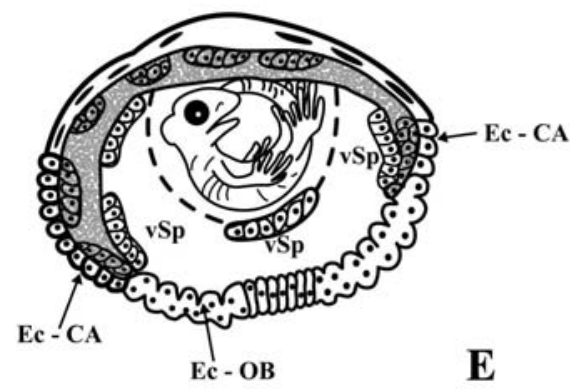

Figura 2. Ectodermo extraembrionario en diferentes estadios de desarrollo. A) Estadios 29-33.

Ectodermo escamoso. (Ca) Calcio, (Ec-OB) Ectodermo de la Onfalopleura Bilaminar,

(MC) Membrana de la Cáscara, 500x. B) Estadio 33. Ectodermo cúbico alto. (Ec-OB) Ectodermo de la Onfalopleura Bilaminar, (MC) Membrana de la cáscara, (SP) esplacnopleura del Saco Vitelino, (U) Útero. 500x. C) Estadio 35. Ectodermo cilíndrico. (Ec-OB) Ectodermo de la Onfalopleura

Bilaminar, (MC) Membrana de la Cáscara. 500x. D) Estadios 36-37. Plegamiento del ectodermo de la

Onfalopleura Bilaminar. (Ec-OB) Ectodermo de la Onfalopleura Bilaminar, (MC) Membrana de la Cáscara, (SV) Saco Vitelino. 200x. E) Esquema de las membranas extraembrionarias en estadio 37.

(Ec-CA) Ectodermo del corioalantoides, (Ec- OB) Ectodermo de la Onfalopleura Bilaminar, (vSp) Vaso de la esplacnopleura. 

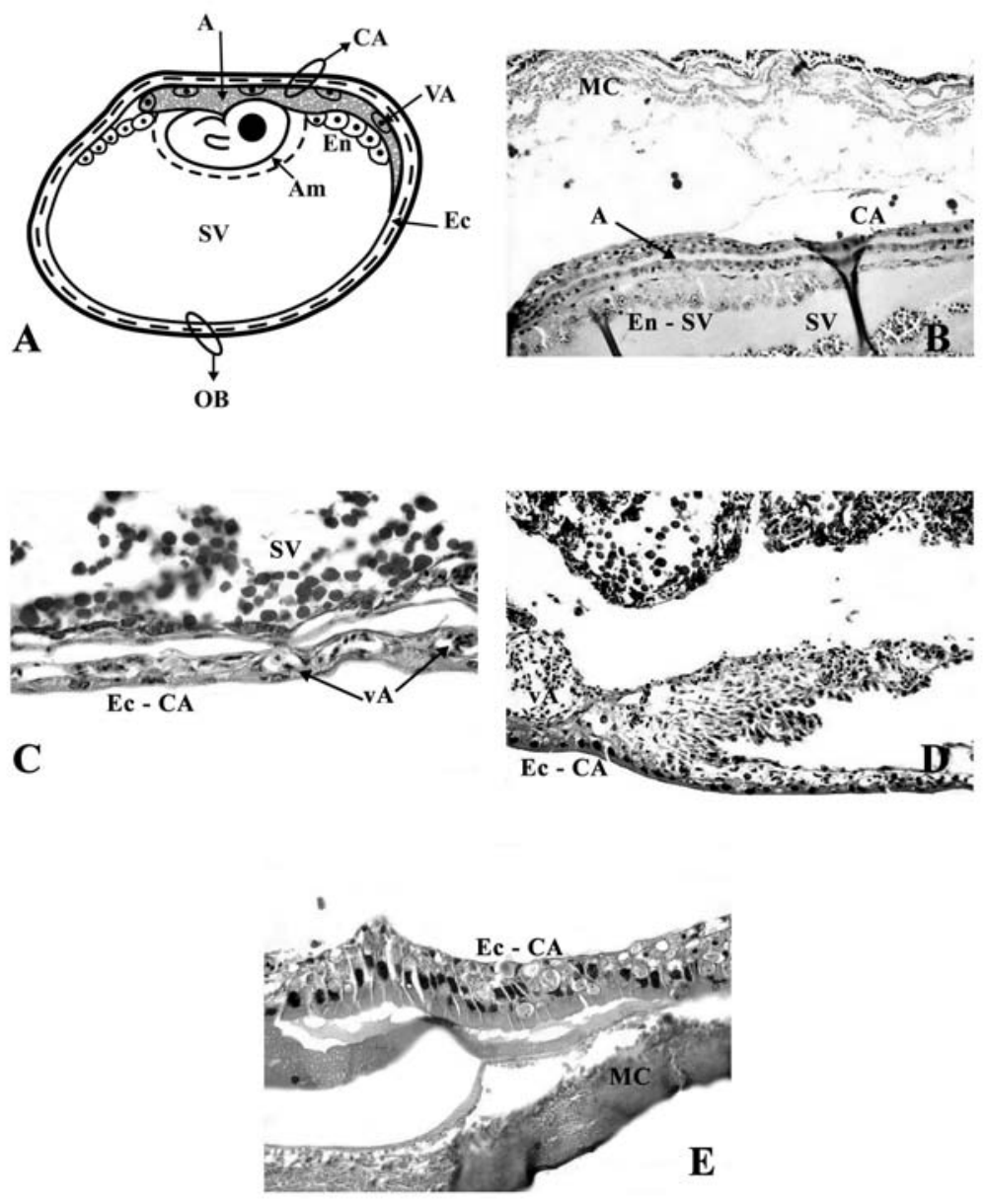

Figura 3. Membranas extraembrionarias en diferentes estadios de desarrollo. A) Esquema de las membranas extraembrionarias en estadio 29. (A) Alantoides, (Am) Amnios, (CA) Membrana Corioalantoidea, (En) Endodermo del Saco Vitelino (OB) Onfalopleura Bilaminar, (VA) Vaso Alantoideo. B) Estadio 29. El alantoides hace contacto con el corion para formar la membrana corioalantoidea sobre una pequeña región del saco vitelino en el hemisferio embrionario.

(CA) Membrana Corioalantoidea, (MC) Membrana de la Cáscara, (A) Alantoides, (En-SV) Endodermo del Saco Vitelino, (SV) Saco Vitelino. 200x. C) Estadios 29-33. Ectodermo escamoso del corioalantoides en una pequeña región del polo abembrionario. (SV) Saco Vitelino, (Ec-CA) Ectodermo del Corioalantoides, (vA) Vaso Alantoideo. 200x. D) Estadio 34. Ectodermo cúbico del corioalantoides en el polo abembrionario. (vA) Vaso Alantoideo, (Ec-CA) Ectodermo del

Corioalantoides. 200x. E) Estadios 35-37. Ectodermo cilíndrico del corioalantoides en el polo abembrionario. (Ec-CA) Ectodermo del corioalantoides, (MC) Membrana de la Cáscara. 500x. 
Saco vitelino. El crecimiento de las células intravitelinas a partir del sinus terminalis, dado por la formación de una delgada masa de vitelo aislada, fue evidente hasta el estadio 31 y estuvieron presentes hasta algunos embriones de estadio 35 . No se encontró evidencia de la formación de una hendidura vitelina.

Membrana corioalantoidea. A partir del estadio 29 de desarrollo embrionario el alantoides ya está presente y hace contacto con el corion para formar la membrana corioalantoidea. Esta membrana se extiende por encima del embrión hasta una pequeña porción del saco vitelino (Figs. 3A y B). La histología reveló que el epitelio coriónico que rodea al alantoides en la región embrionaria del huevo es escamoso, mientras que en el hemisferio abembrionario experimenta diversos cambios estructurales durante la retención, encontrándose un epitelio escamoso del estadio 29-33 (Fig. 3C), cambiando a un epitelio cúbico en el estadio 34 (Fig. 3D) y transformándose a un epitelio cilíndrico en estadios más avanzados (35-37) (Fig. 3E).

En el ectodermo de la membrana corioalantoidea se observaron grandes vesículas de almacén o de secreción. El contenido de estas vesículas se tiñó de manera eosinófila al igual que la membrana de la cáscara (Fig. 3E). Este contenido se hace evidente a partir del estadio 33. La vascularización de la membrana corioalantoidea fue evidente durante la retención intrauterina, incrementándose a partir del estadio 34 (Figs 4A-C).
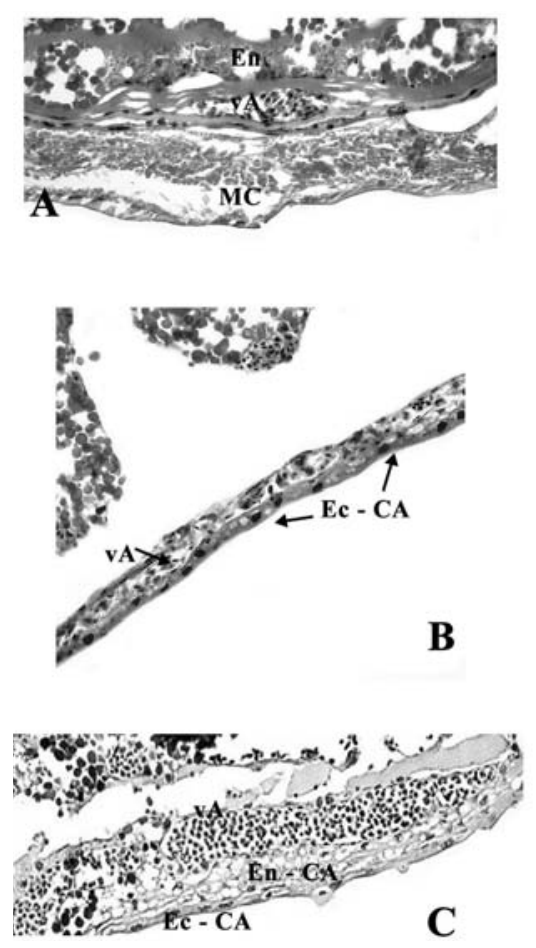

Figura 4. Vascularización de la membrana corioalantoidea en diferentes estadios embrionarios.

A) Estadios 29-33. (vA) Vaso Alantoideo, (MC) Membrana de la Cáscara, (En) Endodermo del Saco Vitelino. 500x. B) Estadios 34-35. (Ec-CA) Ectodermo del Corioalantoides, (vA) Vaso Alantoideo. 200x. C) Estadios 36-37. (Ec-CA) Ectodermo del

Corioalantoides, (En-CA) Endodermo del Corioalantoides, (vA) Vaso Alantoideo. 200x. 
En dos embriones de estadio 34 y 35, la membrana corioalantoidea rodeó por completo al saco vitelino (Fig. 5A y B).

Figura 5. Membrana corioalantoidea en estadios 34-35. A) Esquema del embrión rodeado por la membrana corioalantoidea. (A) Alantoides. (CA) Membrana Corioalantoidea. B) Membrana corioalantoidea en el polo abembrionario rodeando al saco vitelino. (SV) Saco Vitelino, (vA) Vaso Alantoideo, (Ec-CA), Ectodermo del Corioalantoides
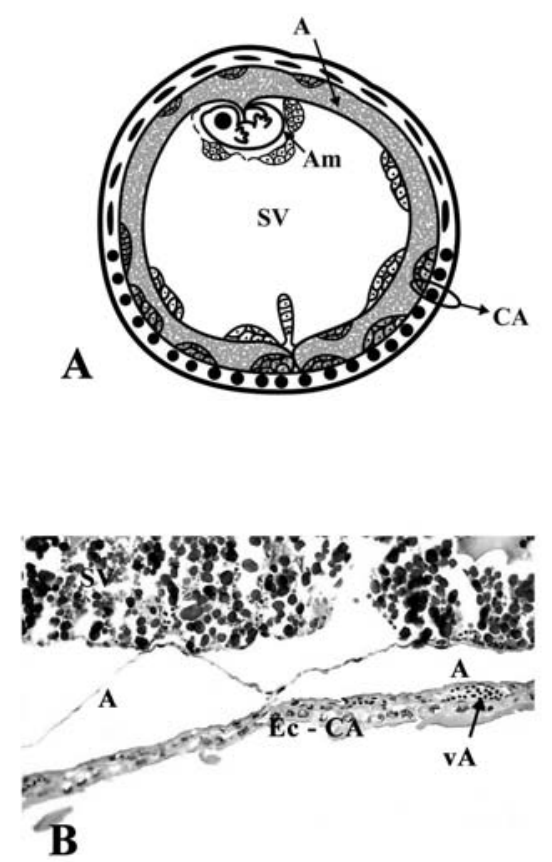

\section{DISCUSIÓN}

La descripción de la ontogenia de las membranas extraembrionarias en escamados ovíparos se ha realizado en Lacerta agilis (Hrabowski 1926), Elgaria multicarinata (Stewart 1985), Bassiana duperreyi (Stewart \& Thompson 1996), Eumeces fasciatus (Stewart \& Florian, 2000), Lacerta vivipara (Stewart \& Thompson 2004) y una culebra ovípara, Elaphe guttata (Blackburn et al. 2003). Estas descripciones permiten hacer una comparación con la ontogenia de estas membranas en S. aeneus.

Membrana coriovitelina. La membrana coriovitelina se forma muy temprano en el desarrollo embrionario y típicamente es transitoria tanto en especies ovíparas como en vivíparas (Blackburn \& Callard 1997, Villagrán et al. 2005). En S. aeneus, esta membrana es sustituida por la membrana corioalantoidea en el estadio 30, mientras que esta membrana en E. fasciatus es reemplazada en el estadio 33 ó 34 (Stewart \& Florian 2000) y en en B. duperreyi ocurre en el estadio 35 (Stewart \& 
Thompson 1996). La histología de la membrana coriovitelina de $S$. aeneus es semejante a la de $B$. duperreyi, donde el ectodermo coriónico es escamoso, a diferencia de lo descrito para E. fasciatus, cuyo ectodermo es cúbico (Stewart \& Florian 2000).

Onfalopleura bilaminar. La histología de la onfalopleura bilaminar de S. aeneus muestra diversos cambios a través del desarrollo embrionario, principalmente en el ectodermo coriónico que lo rodea. En comparación con otras especies ovíparas estudiadas, la onfalopleura bilaminar de E. fasciatus es cúbica y está presente hasta el estadio 37 (Stewart \& Florian, 2000). En B. duperreyi, hay un cambio del ectodermo cúbico a escamoso en el estadio 34 y esta membrana se mantiene hasta el estadio 39 (Stewart \& Thompson 1996). No obstante, el epitelio columnar encontrado en la onfalopleura bilaminar de algunos embriones de los estadios 34 al 36 de $S$. aeneus, fue similar a lo descrito para la especie vivípara Niveoscincus ocellatus (Stewart \& Thompson 2004); además los embriones de estadio 36 de estas dos especies presentaron una onfalopleura bilaminar plegada. Stewart \& Thompson (2004) indicaron que estos pliegues seguramente surgen como resultado de la reducción del vitelo, además de que el área superficial de la onfalopleura de la masa de vitelo aislado no experimenta una reducción simultánea.

Membrana corioalantoidea. El corioalantoides de $S$. aeneus, tiene características muy parecidas a las encontradas en la placenta corioalantoidea de la serpiente vivípara Virginia striatula al mostrar una regionalización en esta membrana (Stewart \& Thompson 2003). En S. aeneus, en los estadios 34 al 37, el ectodermo coriónico de la membrana corioalantoidea en el hemisferio embrionario es escamoso, mientras que la extensión del alantoides en el polo abembrionario, el epitelio coriónico se transforma en cúbico. Todo el epitelio coriónico, presentó grandes vesículas de almacén o de secreción. Stewart \& Thompson (2003), indican que este tipo de tejidos son estructuralmente similares a tejidos de transporte histotrófico.

La característica de que el alantoides rodea por completo al saco vitelino en dos embriones de $S$. aeneus (en estadios 34 y 35), podría estar reflejando las características que se presentan naturalmente en el nido. Sin embargo, es necesario corroborar esta hipótesis con posteriores análisis de las membranas extraembrionarias de huevos en el nido, ya que en este trabajo sólo se enfocó al estudio de dichas membranas durante la retención intrauterina. La característica del alantoides antes descrita, también ha sido observada en algunas especies ovíparas como L. vivipara (en estadios 38-40), E. fasciatus (en estadio 37) y B. duperreyi (en estadio 40). El patrón de crecimiento del alantoides varía entre especies ovíparas; por ejemplo en $L$. vivipara, el alantoides entra por la hendidura vitelina, dejando una pequeña masa de vitelo aislada durante los estadios 38-40 (Stewart \& Thompson, 2004); en E. fasciatus y B. duperreyi, la expansión de esta membrana queda 
determinada por la reabsorción de la masa de vitelo aislada, suplantando a la onfalopleura bilaminar (Stewart \& Florian 2000, Stewart \& Thompson 1996). En $S$. aeneus, a pesar de no tener evidencia de la masa de vitelo aislada en algunos embriones, es posible que el alantoides presente el mismo patrón de crecimiento que en E. fasciatus y B. duperreyi.

Aun cuando este trabajo aporta solamente una descripción histológica de las membranas extraembrionarias, permite concebir una idea general de las posibles funciones que desempeñarían estas membranas durante la retención intrauterina.

La estructura del corioalantoides en el hemisferio abembrionario y la morfología de la onfalopleura bilaminar, hacen que estas membranas se asemejen a tejidos de transporte histotrófico. Si esto es cierto, es posible que algunos elementos tales como iones inorgánicos (calcio) y agua, pasen a través de ellas para ser asimiladas por el embrión. Esto concuerda con los cambios estructurales que experimentan estas membranas en estadios avanzados de desarrollo. Sin embargo, otros análisis, como el rastro de moléculas radiomarcadas, histoquímica enzimática o la interpretación de la ultraestructura celular, serían de gran utilidad para determinar qué tipo de moléculas se transportan a través de las membranas durante la retención intrauterina.

Se ha propuesto que los taxa que han tenido la capacidad de incrementar gradualmente la retención intrauterina, pueden evitar el almacenamiento excesivo de agua en el compartimiento extraembrionario del huevo (Mathies \& Andrews 1996). Es posible que en $S$. aeneus este fenómeno ocurra y que el agua sea asimilada inmediatamente por el embrión con base en sus necesidades fisiológicas dentro del útero. Así, al evitar el incremento en el volumen del huevo, se permite la salida de este a través de la cavidad pélvica de la madre.

La altitud a la que se encuentran sujetos los embriones de S. aeneus $(3200 \mathrm{~m})$ posiblemente influye en el aumento en la densidad vascular de la membrana corioalantoidea a través de la retención, o bien los vasos alantoideos pueden estar participando en la respiración de los embriones que se enfrentan a las condiciones anóxicas del útero. Sin embargo, para probar estas hipótesis, es necesario llevar a cabo un análisis de la estructura y función de las membranas extraembrionarias bajo condiciones normales de anidamiento, así como realizar una comparación de las características de dichas membranas a diferentes elevaciones. Esto permitirá conocer si las modificaciones estructurales que experimentan dichas membranas, principalmente el corioalantoides y la onfalopleura bilaminar, así como el incremento de la vascularización de la membrana corioalantoidea en estadios avanzados de desarrollo, son producto de la retención intrauterina, o bien, son el resultado de la variación altitudinal en la que se distribuye $S$. aeneus. 
AGRADECIMIENTOS. A la Dra. Marcela Aguilar y a la M. en C. Eva Mendoza Cruz de la Facultad de Ciencias, de la UNAM por su asesoría en las técnicas histológicas. A las becas para estudios de maestría del CONACYT, y de tesis del COMECYT, al proyecto de investigación Uaemex 2463/2007 por el apoyo presupuestal para desarrollar la investigación. A la D. G. Misol-ja Méndez por la ilustración y diseño de las figuras. A las personas que participaron en la revisión de este artículo por sus valiosas observaciones.

\section{LITERATURA CITADA}

Andrews, R.M. \& T. Mathies. 2000. Natural history of reptilian development: Constraints on the evolution of viviparity. Bioscience 50(3):227-238.

Andrews, R.M. 1997. Evolution of viviparity: Variation between two sceloporine lizards in the ability to extend egg retention. Journal of Zoology of London. 243: 579-595.

Blackburn, D.G. 1993. Standarized criteria for the recognition of reproductive modes in squamate reptiles. Herpetologica 49:118-132

Blackburn, D.G. \& I.P. Callard. 1997. Morphogenesis of the placental membranes in the viviparous placentotrophic lizards Chalcides chalcides (Squamata: Scincidae). Journal of Morphology 232: 35-55.

Blackburn, D.G., Johnson A.R., \& Petzold J.L. 2003. Histology of the extraembryonic membranes of an oviparous snake: Towards a reconstruction of basal Squamate patterns. Journal of Experimental Zoology 299A:48-58.

Dufaure, J.P. \& J. Hubert. 1961. Table de développement du lézard vivipare: Lacerta (Zooteca) vivipara Jacquin. Archives d'Anatomie Microscopique et de Morphologie Expérimentale 50 : 309-328.

Florian, J.D. Jr. 1990. Development of extraembryonic membranes and chemical composition of eggs and hatchlings of the oviparous lizard, Eumeces fasciatus (Reptilia, Squamata). M.S. Thesis, University of Tulsa, Oklahoma.

Goin, C.J., O.B. Goin, G.R. Zug. 1978. Introduction to Herpetology. Third edition. W.H. Freeman and Company, San Francisco.

Guillette, LJ. Jr. 1993. The evolution of viviparity in lizards. Bioscience 43: 742-751.

Hrabowski, H. 1926. Das dotterorgan der Eidechsen. Zeitschrift fur Wissenschaftliche Zoologie 128: 305-382.

Humason, G.L. 1981. Animal tissue techniques. Second edition. San Francisco: W.H. Freeman.

Mathies, T. \& R.M. Andrews. 2000. Does reduction of the eggshell occur concurrently with or subsequent to evolution of viviparity in phrynosomatid lizards? Biological Journal of the Linnean Society 71: 719-736.

Mathies, T. \& R. M. Andrews. 1996. Extended egg retention and its influence on embryonic development and egg water balance: Implications for the evolution of viviparity. Physiological Zoology 69:1021-1035.

Stewart, J.R. 1985. Placentation in the lizard Gerrhonotus coeruleus with a comparison to the extraembryonic membranes of the oviparous Gerrhonotus multicarinatus (Sauria, Anguidae). Journal of Morphology 185: 101-114.

Stewart, J.R. 1993. Yolk sac placentation in reptiles: Structural innovation in a fundamental vertebrate fetal nutritional system. Journal of Experimental Zoology 266:414-449.

Stewart, J.r. \& J.D. Florian Jr. 2000. Ontogeny of the extraembryonic membranes of the oviparous lizard, Eumeces fasciatus (Squamata: Scincidae). Journal of Morphology 244: 81-107.

Stewart, J.R. \& M.B. Thompson. 1996. Evolution of reptilian placentation: development of extraembryonic membranes of the Australian scincid lizards, Bassiana duperreyi (oviparous) and Pseudemoia entrecasteauxii (viviparous). Journal of Morphology 227:349-370. 
Stewart, J.R. \& M.B. Thompson. 2000. Evolution of placentation among squamate reptiles: recent research and future directions. Comparative Biochemistry and Physiology 127A: 411-431.

Stewart, J.R. \& M.B. Thompson. 2003. Evolutionary transformations of the fetal membranes of viviparous reptiles: a case study of two linages. Journal of Experimental Zoology 299A: 13-32.

Stewart, J.R. \& M.B. Thompson. 2004. Placental ontogeny of the Tasmanian scincid lizard, Niveoscincus ocellatus (Reptilia: Squamata). Journal of Morphology 259: 214-237.

Thompson, M.B., J.R. Stewart \& B.K. Speake. 2000. Comparison of nutrient transport across the placenta of lizards differing in placental complexity. Comparative Biochemistry and Physiology 127A:469-479.

Villagrán, M., F.R. Méndez \& J.R. Stewart. 2005. Placentation in the Mexican lizard Sceloporus mucronatus (Squamata: Phrynosomatidae). Journal of Morphology 264: 286-297. 